\title{
Clinical significance of CCBE1 expression in lung cancer
}

\author{
PENG LI ${ }^{1}$, ZHUANGZHUANG CONG $^{2}$, YONG QIANG ${ }^{1,2}$, LEI XIONG $^{1,2}$, LI TANG $^{2}$, YU ZHANG $^{3}$, \\ HAIWEI WU ${ }^{1,2}$, JUN YI ${ }^{1,2}$, HUA JING ${ }^{1,2}$, DEMIN LI ${ }^{1,2}$ and YI SHEN ${ }^{1,2}$ \\ ${ }^{1}$ Department of Cardiothoracic Surgery, Jinling Hospital, Southern Medical University, Guangzhou, Guangdong 510515; \\ ${ }^{2}$ Department of Cardiothoracic Surgery, Jinling Hospital, Medical School of Nanjing University, Nanjing, Jiangsu 210002; \\ ${ }^{3}$ Department of Laboratory Medicine of Jiangsu University, Zhenjiang, Jiangsu 212013, P.R. China
}

Received March 8, 2016; Accepted February 22, 2017

DOI: $10.3892 / \mathrm{mmr} .2017 .8187$

\begin{abstract}
Lymph node metastasis (LNM) is one of the major causes of cancer-associated morbidity and mortality in patients with lung cancer following radical pulmonary carcinoma resection. The present study aimed to investigate the relationship between the expression of collagen and calcium-binding epidermal growth factor domain-containing protein 1 (CCBE1) and lymphatic vessel endothelial hyaluronan receptor 1 (LYVE1) in tumor tissue with the clinical prognosis of lung cancer. The present study included 40 patients with lung cancer that underwent pulmonary carcinoma resection, including 10 patients with LNM, and 10 control patients who underwent pulmonary bullae resection. CCBE1 and LYVE1 expression was assessed in samples from normal and tumor tissue using polymerase chain reaction, western blot analysis and immunohistochemistry. CCBE1 expression appeared to be decreased in lung tumor tissue and further downregulated in samples from patients with LNM, and was revealed to be correlated with poor clinical outcome. Conversely, LYVE1 expression appeared to be upregulated in lung cancer tissue. In conclusion, the present results suggested that CCBE1 and LYVE1 may have potential as biomarkers for the identification of lung cancer patients at a high risk of LNM.
\end{abstract}

\section{Introduction}

Lung cancer is the leading cause of cancer-associated mortality in the Chinese population. In 2015, an estimated 4292,000 new cancer cases and 2814,000 cancer deaths occurred in China, with lung cancer being the most common cancer and the leading cause of cancer death (1). Lung cancer

Correspondence to: Professor Yi Shen, Department of Cardiothoracic Surgery, Jinling Hospital, Medical School of Nanjing University, 305 East Zhongshan Road, Nanjing, Jiangsu 210002, P.R. China

E-mail: yi_shen12@sina.com

Key words: collagen and calcium binding EGF domains 1, lymphatic vessel endothelial hyaluronan receptor 1 , lymph node metastasis, lung cancer is among the most common types of cancer, followed by stomach, colorectal, liver and esophageal cancer. In 2015, lung cancer was the most frequent type of cancer in male, followed by stomach, liver, colorectal and esophageal cancer; among females, breast cancer was the most frequent type of cancer, followed by lung, colorectal, stomach and liver cancer (1-3). Therefore, the identification of potential biomarkers for the early diagnosis and prognosis of lung cancer is imperative.

Collagen and calcium-binding epidermal growth factor domain-containing protein (CCBE) 1 has been identified as a novel lymphangiogenic signaling factor in zebrafish (4). Mutations in CCBE1 have been reported to cause thoracic duct and dorsal lymphatic vessel malformation; however, they had no effect on blood vasculature (5). During embryogenesis, CCBE1 and vascular endothelial growth factor-C (VEGF-C) were demonstrated to stimulate angiogenic sprouting and lymphangiogenic budding from the venous endothelium (4). CCBE1 was revealed to be necessary for lymphangiogenesis; however, it was not identified as a component of the VEGF-C/VEGF receptor 3 (VEGFR3) signaling pathway or the sex determining region Y-box 18 (SOX18)/prospero homeobox protein 1 (Prox1) transcriptional pathway (6). Therefore, it was suggested that CCBE1 may function as an independent regulator of lymphangioblast budding and, possibly, migration (7). CCBE1 mutations in zebrafish, similar to VEGF-C, Prox1 and SOX18 mutations, were demonstrated to cause specific loss of embryonic lymphatic vasculature (7).

CCBE1-deficient mouse embryos were reported to lack lymphatic structures, thus suggesting a critical role for CCBE1 in lymphangiogenesis (6). In addition, CCBE1 has been identified as one of the causal genes that may be responsible for primary generalized lymph vessel dysplasia associated with the autosomal recessive Hennekam syndrome in humans, which is associated with lymphedema, lymphangiectasia, mental retardation and unusual facial characteristics. It has been suggested that CCBE1 mutations may cause a similar phenotype, thus making CCBE1 a candidate gene implicated in human generalized lymphatic dysplasia (8).

It has previously been reported that in zebrafish CCBE1 may interact genetically with VEGF-C and VEGFR3 (4), whereas a VEGF-C mutation has been implicated in inherited lymphedema (9). CCBE1 has been demonstrated to function during the same developmental stages as VEGF-C to promote lymphatic sprouting; however, the biochemical roles of CCBE1 
in lung cancer have yet to be elucidated (5). CCBE1 has been suggested to function as a tumor suppressor in ovarian cancer, since low CCBE1 gene expression has been associated with the degree of tumor differentiation and the pathologic stage of the disease, as well as with a low survival rate (8). Furthermore, it has been demonstrated that CCBE1 expression levels were downregulated in laryngocarcinoma, thus suggesting that CCBE1 may have potential as a novel biomarker for the estimation of laryngeal cancer prognosis (10). In addition, evaluation of the lymphatic expression of CCBE1 may also have potential use in colorectal cancer prognosis (11). Moreover, a positive association between the expression of CCBE1 and LYVE1 was reported in lung cancer (12). The present study investigated the expression of CCBE1 and lymphatic vessel endothelial hyaluronan receptor 1 (LYVE1) in tissue samples from patients with lung cancer, to evaluate the potential of CCBE1 as a biomarker for the diagnosis of lung cancer.

\section{Materials and methods}

Patients. A total of 50 patients, including 40 patients with lung cancer and 10 patients with pulmonary bullae, were enrolled in the present study between May and July 2013 in Jinling Hospital (Nanjing, China). Among the patients with lung cancer, 10 patients exhibited evidence of lymph node metastasis (LNM) and 30 patients did not. The patients did not receive any preoperative anticancer treatment. Patients with lung cancer underwent pulmonary carcinoma resection, whereas control patients underwent pulmonary bullae resection. The study population consisted of 30 males and 20 females with a mean age of 53.35 years (range, 45-64 years). The lung tissues were obtained from the lung bullae from lung cancer patients and normal controls.

The present study was approved by the Ethics Committee of Southern Medical University (Guangzhou, China). Written informed consent was obtained from all human subjects prior to enrollment in the present study.

Reverse transcription-polymerase chain reaction (RT-PCR). Total RNA was extracted from tissue samples isolated from patients with lung cancer or pulmonary bullae using TRIzol ${ }^{\circledR}$ reagent (Invitrogen; Thermo Fisher Scientific, Inc., Waltham, MA, USA) according to the manufacturer's instructions. Total RNA $(2 \mu \mathrm{g})$ was reverse transcribed (20 $\mu 1$ reaction volume) into cDNA using a reverse transcription system, as previously described (13). The following primers, designed using the Primer Premier software version 5.0 (Premier Biosoft International, Palo Alto, CA, USA) and synthesized by Sangon Biotech Co., Ltd. (Shanghai, China) were employed: CCBE1 forward, 5'-CGA CTAAATACCCGTGTCTGAAG-3' and reverse, 5'-TCGGCA CAAACGTCGTAATCT-3'; $\beta$-actin forward, 5'-GCTCGTCGT CGACAACGGCTC-3' and reverse, 5'-CAAACATGATCT GGGTCACTTCTC-3'. Amplification was performed under the following conditions: Initial incubation at $95^{\circ} \mathrm{C}$ for $10 \mathrm{sec}$, followed by 40 cycles at $95^{\circ} \mathrm{C}$ for $5 \mathrm{sec}$ and at $62^{\circ} \mathrm{C}$ for $45 \mathrm{sec}$, and extension at $72^{\circ} \mathrm{C}$ for $3 \mathrm{~min}$. The $25-\mu$ LIPCR reaction system included $1 \mu \mathrm{l}$ temple, $2 \mu \mathrm{l}$ primers, $2 \mu \mathrm{ldNTP}, 0.5 \mu \mathrm{l}$ RT/Platinum ${ }^{\mathrm{TM}}$ Taq Mix (Invitrogen; Thermo Fisher Scientific, Inc.) and distilled water. PCR products were detected by $2 \%$ agarose gel electrophoresis as previously described (13).
Western blot analysis. The protein was extracted by radioimmunoprecipitation assay buffer cell lysis (containing PMSF) (Invitrogen; Thermo Fisher Scientific, Inc.). Protein concentration was quantified using BCA protein assay kit (Beyotime Institute of Biotechnology, Shanghai, China) $20 \mu \mathrm{g}$ protein were separated by $10 \%$ SDS-PAGE and transferred onto nitrocellulose membranes. The membranes were blocked by 5\% skimmed milk in PBST (1\% Tween-20) at room temperature for $2 \mathrm{~h}$. Membranes were probed with anti-CCBE1 (1:1,000, cat. no. HPA041361; Sigma-Aldrich; Merck KGaA, Darmstadt, Germany), anti-LYVE1 (1:1,000, cat. no. ab10278; Abcam, Shanghai, China) and anti- $\beta$-actin $(1: 5,000$, cat. no. ab8227; Abcam) primary antibodies at $4^{\circ} \mathrm{C}$ overnight. Subsequently, membranes were incubated with horseradish peroxidase-conjugated aoat anti-rabbit immunoglobulin G secondary antibodies (1:10,000, cat. no. TA130023; OriGene Technologies, Inc., Beijing, China) at room temperature for $2 \mathrm{~h}$. Protein bands were visualized using an enhanced chemiluminescence detection kit. The blots were semi-quantified by densitometry analysis using the Multi-Analyst ${ }^{\circledR}$ software version 2.0.1 (Bio-Rad Laboratories, Inc., Hercules, CA, USA).

Immunohistochemistry. Immunohistochemical staining was performed using a two-step method with heat-induced antigen-retrieval procedures (14). The cancer tissues were fixed in $4 \%$ paraformaldehyde. Following dehydration, the tissue was sliced and blocked in 10\% BSA (Sigma-Aldrich; Merck KGaA). The following primary antibodies were used: Rabbit anti-human CCBE1 monoclonal antibody (cat. no. HPA041361, 1:50-1:200; Sigma-Aldrich, Merck KGaA) and rabbit anti-human LYVE1 monoclonal antibody (cat. no. ab33682, 1:100; Abcam). The slices $(20 \mu \mathrm{m})$ were incubated with horseradish peroxidase-conjugated secondary antibodies (1:100, peroxidase conjugated goat anti-rabbit IgG, TA130023; OriGene Technologies, Inc.) at room temperature for $2 \mathrm{~h}$. The primary antibody was applied in the negative control. The images were visualized by light microscope (Olympus Corporation, Tokyo, Japan).

Statistical analysis. Data are expressed as the mean \pm standard deviation SPSS software was used for analysis (version 17.0; SPSS, Inc., Chicago, IL, USA). One-way analysis of variance was used to analyze the difference among three or more groups followed by Tukey's test. $\chi^{2}$ analysis was used to assess disease recurrence in patients with or without LNM. The association of LNM and disease recurrence over time was confirmed by Kaplan-Meier analysis. $\mathrm{P}<0.05$ was considered to indicate a statistically significant difference.

\section{Results}

CCBE1 and LYVE1 expression in lung cancer tissue. RT-PCR analysis indicated that the mRNA expression levels of CCBE1 were reduced in lung cancer tissue compared with normal tissue (pulmonary bullae). Furthermore, tissue samples from patients with LNM exhibited reduced CCBE1 mRNA expression levels compared with in samples from patients with cancer without LNM (Fig. 1A). CCBE1 protein expression levels exhibited a similar trend, as revealed using western blot analysis (Fig. 1B). Conversely, the protein expression levels 
A

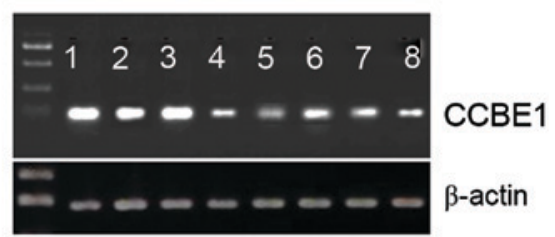

B

$\begin{array}{llllllll}1 & 2 & 3 & 4 & 5 & 6 & 7 & 8\end{array}$

C

$\begin{array}{llllllll}1 & 2 & 3 & 4 & 5 & 6 & 7 & 8\end{array}$

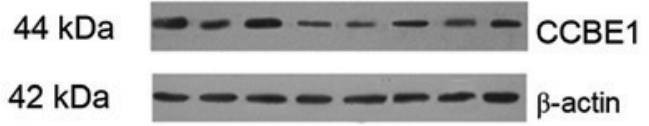

$35 \mathrm{kDa}$

- - - - - - - LYVE1

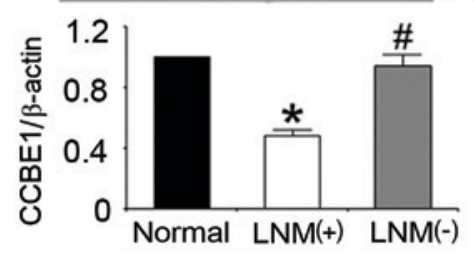

$42 \mathrm{kDa}$
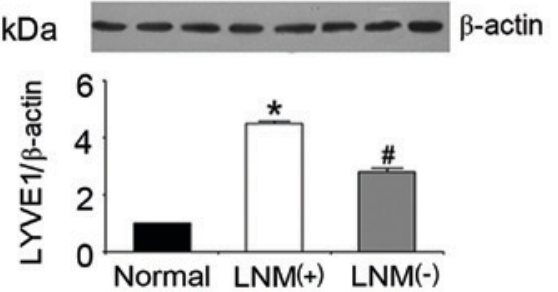

Figure 1. CCBE1 and LYVE1 expression in patients with lung cancer and healthy controls. (A) Reverse transcription-polymerase chain reaction analysis of CCBE1 mRNA expression levels in lung tissue samples. Western blot analysis of (B) CCBE1 and (C) LYVE-1 protein expression levels in lung tissue samples. Lanes 1-3 represent samples from normal tissue; lanes 4 and 5 represent samples from patients with cancer and LNM; lanes 6-8 represent samples from patients with cancer without LNM. Data are expressed as the mean \pm standard deviation ( $\mathrm{n}=5$ in each group). " $\mathrm{P}<0.05$ vs. normal group; ${ }^{\#} \mathrm{P}<0.05$ vs. LNM group. CCBE1, collagen and calcium-binding epidermal growth factor domain-containing protein 1; LYVE1, lymphatic vessel endothelial hyaluronan receptor 1; LNM, lymph node metastasis.

A

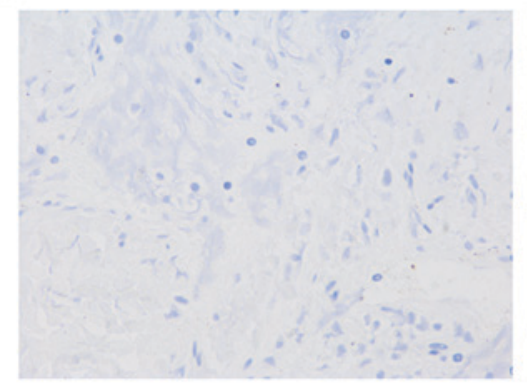

C

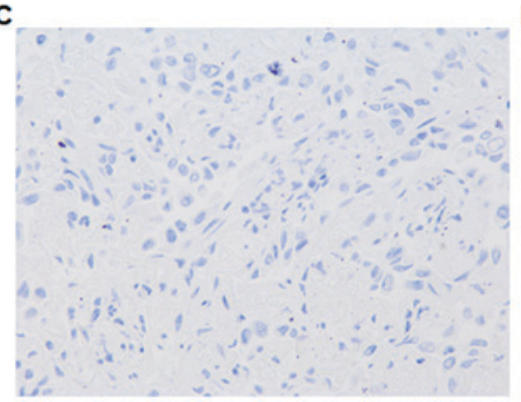

B

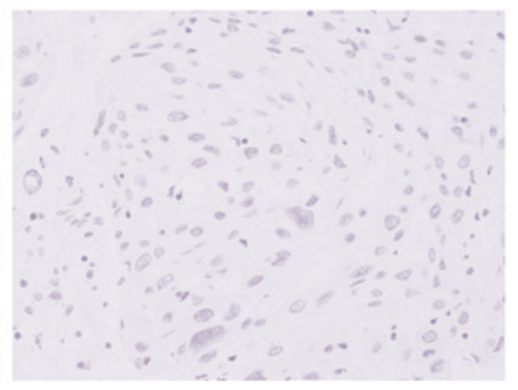

D

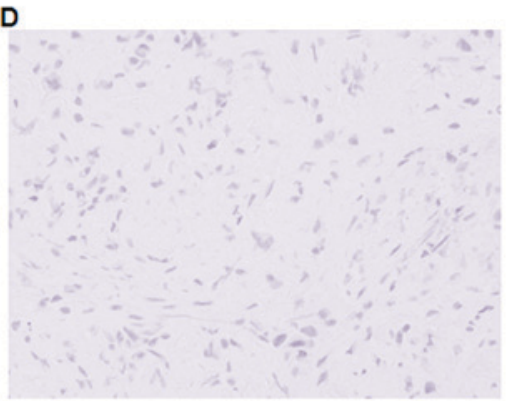

Figure 2. CCBE1 expression in patients with lung cancer and healthy controls. Representative CCBE1 immunostaining from is demonstrated. (A) Negative control. (B) Tissue isolated from control patients who underwent pulmonary bullae resection. (C) Tissue isolated from patients with lung cancer without LNM. (D) Tissue isolated from patients with lung cancer accompanied by LNM. Photomicrographs were captured under x400 magnification. CCBE, collagen and calcium-binding epidermal growth factor domain-containing protein; LNM, lymph node metastasis.

of the lymphatic-specific marker LYVE1 were revealed to be significantly upregulated in tissue samples from patients with lung cancer compared with in normal tissue samples. Furthermore, LYVE1 levels were significantly increased in samples from patients with LNM compared with patients without LNM (Fig. 1C).

Consistent with the immunoblotting data, immunohistochemical analysis indicated that CCBE1 expression appeared to be downregulated in tissue samples from patients with lung cancer compared with in normal tissue samples. In addition, patients with LNM appeared to exhibit reduced CCBE1 expression compared with patients with cancer without LNM (Fig. 2). LYVE1 appeared to be expressed at higher levels in lung cancer samples compared with in normal tissue samples; LYVE1 appeared to be upregulated in samples from patients with LNM compared with in patients without LNM (Fig. 3). Thus, there was an inverse association between expression of CCBE1 and LYVE-1 in normal and tumor-derived tissues. 

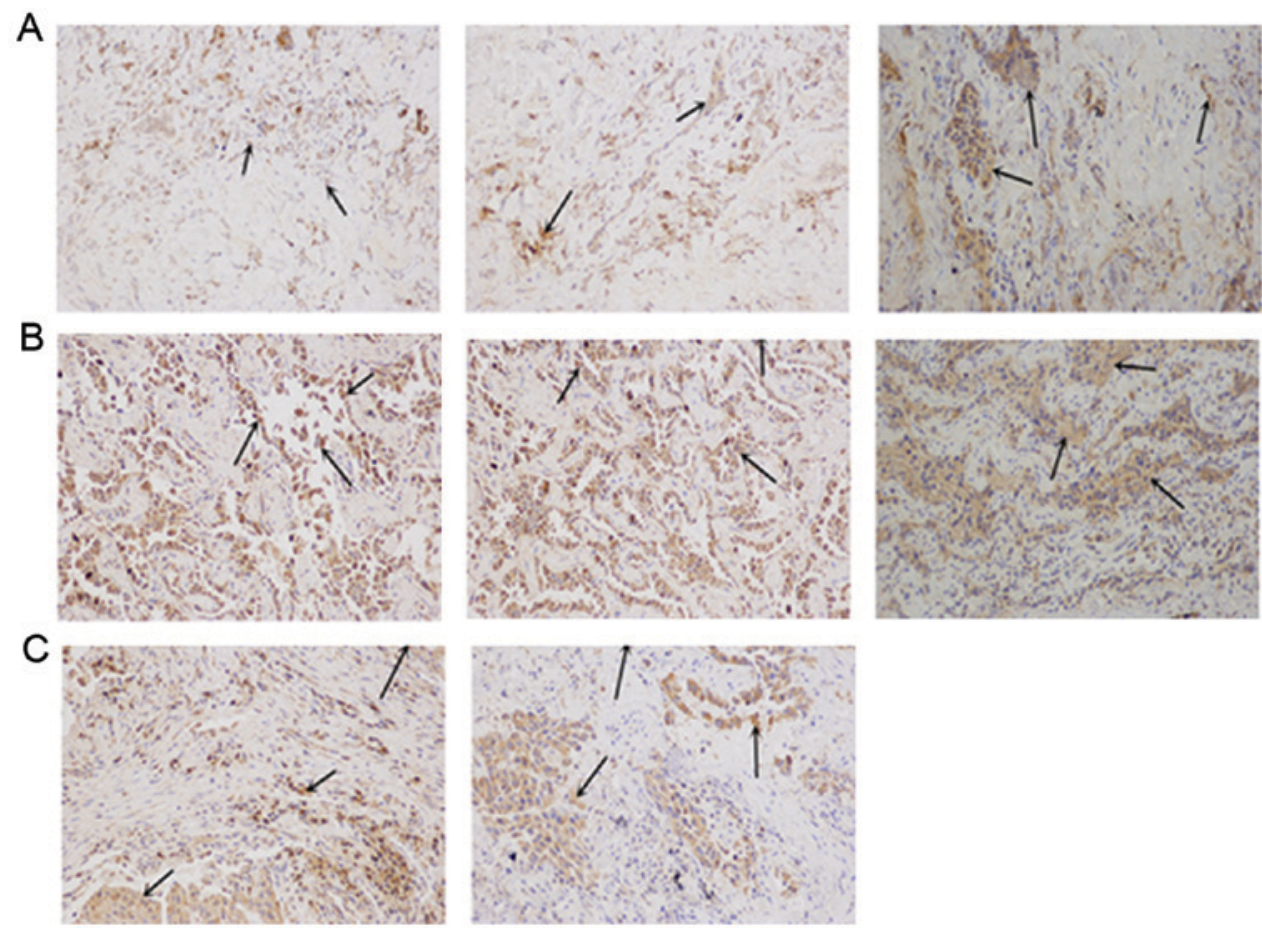

Figure 3. LYVE1 expression in patients with lung cancer and healthy controls. Representative cytoplasmic LYVE1 staining from a total of 8 patients is demonstrated. (A) Tissues isolated from control patients who underwent pulmonary bullae resection. (B) Tissues isolated from patients with lung cancer without LNM. (C) Tissues isolated from patients with lung cancer accompanied by LNM. Black arrows indicate LYVE1 staining patterns. Photomicrographs were captured under x200 magnification. LYVE, lymphatic vessel endothelial hyaluronan receptor; LNM, lymph node metastasis.

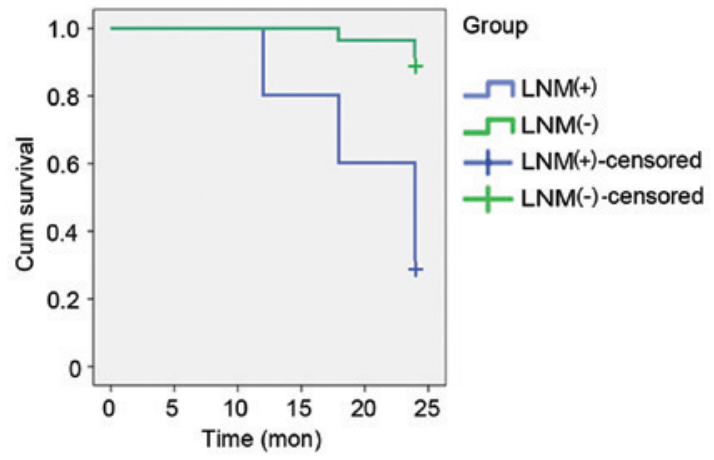

Figure 4. Kaplan-Meier analysis of the overall survival of patients with lung cancer, with or without LNM. ( $\mathrm{n}=5$ in each group). LNM, lymph node metastasis.

Association between CCBE1 expression and disease prognosis. Patients with lung cancer that were enrolled in the present study were followed up at 6-month intervals for 2 years. No significant differences in clinical prognosis were revealed 6 months after pulmonary carcinoma resection among patients with or without LNM; however, 12 months after surgery, two patients with LNM exhibited recurrent lung cancer. A total of 18 months after surgery, 2 patients with LNM exhibited disease recurrence, as well as 1 patient without LNM. By 24 months, an additional 3 patients with LNM exhibited disease recurrence, as well as an additional 2 patients without LNM. $\chi^{2}$ analysis of the proportion of disease recurrence in patients with or without LNM revealed significantly higher rates of recurrence among patients with $\mathrm{LNM}(\mathrm{P}<0.05)$ at 12,18 and 24 months post-surgery
Table I. Results of $\chi^{2}$ analysis of cancer recurrence ( $\mathrm{n}=40$ patients).

\begin{tabular}{lcccc}
\hline $\begin{array}{l}\text { Time post-surgery } \\
\text { (months) }\end{array}$ & $\begin{array}{c}\text { Group A } \\
\text { LNM (+) }\end{array}$ & $\begin{array}{c}\text { Group B } \\
\text { LNM (-) }\end{array}$ & $\begin{array}{c}\chi^{2} \\
\text { value }\end{array}$ & P-value \\
\hline 12 & & & 6.32 & 0.012 \\
$\quad$ Recurrence & 2 & 0 & & \\
$\quad$ No recurrence & 8 & 30 & & \\
18 & & & 9.22 & 0.002 \\
Recurrence & 4 & 1 & & \\
No recurrence & 6 & 29 & & \\
24 & & & 14.40 & $<0.01$ \\
Recurrence & 7 & 3 & & \\
No recurrence & 3 & 27 & & \\
\hline
\end{tabular}

LNM (+), patients with lung cancer and LNM; LNM (-), patients with lung cancer without LNM; LNM, lymph node metastasis.

$\left(\chi^{2}=6.32,9.22\right.$ and 14.4 , respectively; Table I). Prior to 12 months, no significant differences were observed. The association between LNM and disease recurrence over time was confirmed by Kaplan-Meier analysis (Fig. 4).

\section{Discussion}

In the present study, mRNA and protein expression levels of CCBE1 and LYVE1 were investigated in patients with lung cancer. CCBE1 expression was revealed to be downregulated 
in lung cancer tissue. Notably, tumor tissue isolated from patients with LNM exhibited the lowest CCBE1 expression. Conversely, LYVE1 expression was revealed to be upregulated in tissue samples isolated from patients with lung cancer; similarly, its expression appeared to be highest in tissue samples form patients with LNM. These results suggested that CCBE1 may have potential as a diagnostic biomarker for lung cancer and LNM.

Previous studies have suggested that CCBE1 may participate in extracellular matrix remodeling and cellular migration (15). CCBE1 mutations have been reported to cause lymphatic dysplasia, lymphedema-cholestasis syndrome and fetal hydrops $(16,17)$. In ovarian cancer cell lines and primary carcinomas, CCBE1 appeared to be downregulated, thus suggesting that it may serve a role as a tumor suppressor (15). In the present study, CCBE1 expression was revealed to be downregulated in tissue samples from patients with lung cancer. Notably, samples isolated from patients with lung cancer accompanied by LNM exhibited the lowest CCBE1 expression levels, thus suggesting that lung cancer progression may be associated with a decrease in CCBE1 expression.

The collagen domains in the CCBE1 structure are implicated in the activation of VEGF-C (18), which is a critical molecule for angiogenesis and tumor metastasis (19). LYVE1 acts as a receptor for hyaluronan and binds to the soluble and immobilized form of the protein. It has previously been suggested that LYVE1 may participate in lymphatic hyaluronan transport and serve a role in tumor metastasis (20). The evolutionary conservation of LYVE1 suggests an important role for this protein in lymph node metastasis (21); however, its physiological role has yet to be elucidated. It has previously been demonstrated that immunocytochemical staining for LYVE1 was able to recognize lymphatic vessel endothelium and pancreatic endocrine cells (22). In the present study, LYVE1 protein expression levels were significantly upregulated in patients with lung cancer, particularly among patients with LNM.

The present results suggested that CCBE1 expression was downregulated, whereas LYVE1 expression was upregulated in lung cancer tissue. Notably, a previous study reported a positive correlation between the expression of CCBE1 and LYVE1 (12). However, the positive correlation has been established in normal tissue. Therefore, it may be hypothesized that in cancer tissue, CCBE1 downregulation can stimulate LYVE1 to promote LNM.

The present study demonstrated the association between LNM and disease recurrence. The present findings revealed a direct correlation between CCBE1 expression and disease recurrence, suggesting that reduced $\mathrm{CCBE} 1$ expression may be associated with an increased risk of disease recurrence in patients with lung cancer. Therefore, the expression of CCBE1 in tumor tissue from lung cancer patients may have potential as a biomarker for the prognosis of lung cancer and for evaluating the risk of LNM.

In conclusion, the present results demonstrated that CCBE1 expression was downregulated in lung cancer, particularly in the presence of LNM. Therefore, it may be hypothesized that CCBE1 has potential as a biomarker to evaluate lung cancer prognosis and assess the risk of lymph node metastasis.

\section{Acknowledgements}

The present study was supported by the National Natural Science Foundation of China (grant no. 81172032).

\section{References}

1. Chen W, Zheng R, Baade PD, Zhang S, Zeng H, Bray F, Jemal A, $\mathrm{Yu}$ XQ and He J: Cancer statistics in China, 2015. CA Cancer J Clin 66: 115-132, 2016.

2. Chen W, Zheng R, Zhang S, Zhao P, Li G, Wu L and He J: Report of incidence and mortality in China cancer registries, 2009. Chin J Cancer Res 25: 10-21, 2013.

3. Parkin DM, Pisani P, Lopez AD and Masuyer E: At least one in seven cases of cancer is caused by smoking. Global estimates for 1985. Int J Cancer 59: 494-504, 1994.

4. Le Guen L, Karpanen T, Schulte D, Harris NC, Koltowska K, Roukens G, Bower NI, van Impel A, Stacker SA, Achen MG, et al: Ccbe1 regulates Vegfc-mediated induction of Vegfr3 signaling during embryonic lymphangiogenesis. Development 141: 1239-1249, 2014.

5. Stacker SA, Williams SP, Karnezis T, Shayan R, Fox SB and Achen MG: Lymphangiogenesis and lymphatic vessel remodelling in cancer. Nat Rev Cancer 14: 159-172, 2014.

6. Bos FL, Caunt M, Peterson-Maduro J, Planas-Paz L, Kowalski J, Karpanen T, van Impel A, Tong R, Ernst JA, Korving J, et al: CCBE1 is essential for mammalian lymphatic vascular development and enhances the lymphangiogenic effect of vascular endothelial growth factor-C in vivo. Circ Res 109: 486-491, 2011.

7. Hogan BM, Bos FL, Bussmann J, Witte M, Chi NC, Duckers HJ and Schulte-Merker S: Ccbe1 is required for embryonic lymphangiogenesis and venous sprouting. Nat Genet 41: 396-398, 2009.

8. Alders M, Hogan BM, Gijini E, Salehi F, Al-Gazali L, Hennekam EA, Holmberg EE, Mannens MM, Mulder MF, Offerhaus GJ, et al: Mutations in CCBE1 cause generalized lymph vessel dysplasia in humans. Nat Genet 41: 1272-1274, 2009.

9. Gordon K, Schulte D, Brice G, Simpson MA, Roukens MG, van Impel A, Connell F, Kalidas K, Jeffery S, Mortimer PS, et al: Mutation in vascular endothelial growth factor-C, a ligand for vascular endothelial growth factor receptor-3, is associated with autosomal dominant milroy-like primary lymphedema. Circ Res 112: 956-960, 2013.

10. Wang Y, Li W, Zhang D, Kang S, Ding L and Zhao S: The expression and clinical significance of CCBE1 in throat cancer. J Shanxi Med Uni 43: 58-61, 2012.

11. Li W, Wang Y, Liu Y, Yin G and Feng K: Prognosis of CCBE1 expression in colorectal cancer. Chin J Gerontol 7: 3849-3850, 2014.

12. Jeltsch M, Jha SK, Tvorogov D, Anisimov A, Leppänen VM, Holopainen T, Kivelä R, Ortega S, Kärpanen T and Alitalo K: CCBE1 enhances lymphangiogenesis via A disintegrin and metalloprotease with thrombospondin motifs-3-mediated vascular endothelial growth factor-C activation. Circulation 129: 1962-1971, 2014.

13. Zhu G, Li J, He L, Wang X and Hong X: MPTP-induced changes in hippocampal synaptic plasticity and memory are prevented by memantine through the BDNF-TrkB pathway. Br J Pharmacol 172: 2354-2368, 2015.

14. Wu S, Cao J, Zhang T, Zhou Y, Wang K, Zhu G and Zhou M: Electroacupuncture ameliorates the coronary occlusion related tachycardia and hypotension in acute rat myocardial ischemia model: Potential role of hippocampus. Evid Based Complement Alternat Med 2015: 925987, 2015.

15. Barton CA, Gloss BS, Qu W, Statham AL, Hacker NF, Sutherland RL, Clark SJ and O'Brien PM: Collagen and calcium-binding EGF domains 1 is frequently inactivated in ovarian cancer by aberrant promoter hypermethylation and modulates cell migration and survival. Br J Cancer 102: 87-96, 2010.

16. Connell FC, Kalidas K, Ostergaard P, Brice G, Murday V, Mortimer PS, Jeffrey I, Jeffery S and Mansour S: CCBE1 mutations can cause a mild, atypical form of generalized lymphatic dysplasia but are not a common cause of non-immune hydrops fetalis. Clin Genet 81: 191-197, 2012.

17. Shah S, Conlin LK, Gomez L, Aagenaes $\varnothing$, Eiklid K, Knisely AS, Mennuti MT, Matthews RP, Spinner NB and Bull LN: CCBE1 mutation in two siblings, one manifesting lymphedema-cholestasis syndrome, and the other, fetal hydrops. PLoS One 8: e75770, 2013. 
18. Roukens MG, Peterson-Maduro J, Padberg Y, Jeltsch M Leppänen VM, Bos FL, Alitalo K, Schulte-Merker S and Schulte D: Functional dissection of the CCBE1 protein: A crucial requirement for the collagen repeat domain. Circ Res 116: $1660-1669,2015$

19. Liu HT, Xing AY, Chen X, Ma RR, Wang YW, Shi DB, Zhang H, Li P, Chen HF, Li YH and Gao P: MicroRNA-27b, microRNA-101 and microRNA-128 inhibit angiogenesis by down-regulating vascular endothelial growth factor $\mathrm{C}$ expression in gastric cancers. Oncotarget 6: 37458-37470, 2015.

20. Jackson DG: The lymphatics revisited: New perspectives from the hyaluronan receptor LYVE-1. Trends Cardiovasc Med 13: $1-7,2003$.
21. Ramani P, Dungwa JV and May MT: LYVE-1 upregulation and lymphatic invasion correlate with adverse prognostic factors and lymph node metastasis in neuroblastoma. Virchows Arch 460: 183-191, 2012.

22. Tomita T: Lymphatic vessel endothelial hyaluronan receptor 1 immunocytochemical staining for pancreatic islets and pancreatic endocrine tumors. Pancreas 35: e18-e22, 2007.

cc) (i) (-) This work is licensed under a Creative Commons

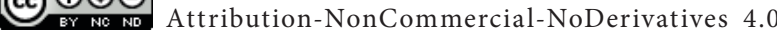
International (CC BY-NC-ND 4.0) License. 